\title{
Hypoxia-Induced TPM2 Methylation is Associated with Chemoresistance and Poor Prognosis in Breast Cancer
}

\author{
Jinfeng Zhang ${ }^{\mathrm{a}} \quad$ Jian Zhang ${ }^{\mathrm{a}}$ Shouping $\mathrm{Xu}^{\mathrm{a}} \quad$ Xianyu Zhang ${ }^{\mathrm{a}}$ Peiyuan Wang ${ }^{\mathrm{a}}$ \\ Hao Wu ${ }^{a}$ Bingshu Xia ${ }^{a}$ Guangwen Zhang ${ }^{a}$ Bo Lei ${ }^{a}$ Lin Wan ${ }^{a}$ Dekai Zhang ${ }^{a, b}$ \\ Da Pang ${ }^{\mathrm{a}, \mathrm{c}}$
}

aDepartment of Breast Surgery, Harbin Medical University Cancer Hospital, Harbin, ${ }^{b}$ Department of

Gastroenterology, The 2nd Affiliated Hospital of Harbin Medical University, Harbin, cHeilongjiang

Academy of Medical Sciences, Harbin, China

\section{Key Words \\ Tpm2 • Hypoxia • Methylation • Breast cancer • Paclitaxel}

\begin{abstract}
Background/Aims: Tropomyosin-2 (TPM2) plays important roles in functions of the cytoskeleton, such as cytokinesis, vesicle transport, cell proliferation, migration and apoptosis, and these functions imply that TPM2 also plays a role in cancer development. Indeed, it has been shown that TPM2 plays a critical role in some cancers. However, the role of TPM2 in breast cancer is still poorly characterized. Thus, we explored the role of TPM2 in breast cancer. Methods: We analysed TPM2 expression and its correlation with the clinicopathological features in breast cancer. Then, we examined the influence of hypoxia on TPM2 expression and methylation status using bisulfite sequencing PCR. Furthermore, we performed TPM2mediated migration and invasion assays in the context of hypoxia and examined changes in matrix metalloproteinase-2 (MMP2) expression. Finally, we detected the influence of TPM2 on survival and chemotherapy drug sensitivity. Results: We found that TPM2 expression is down-regulated in breast cancer cells compared to that in normal breast cells. The data from TCGA supported these results. Promoter methylation of TPM2, which could be induced by hypoxia, was responsible for its low expression. Hypoxia might regulate cell invasiveness partly by TPM2 down-regulation-mediated changes of MMP2 expression. Importantly, low TPM2 expression was correlated with lymph node metastasis $(P=0.031)$, tumour node metastasis stage $(P=0.01)$, histological grade $(P=0.037)$, and shorter overall survival $(P=0.028)$. Univariate and multivariate analyses indicated that TPM2 was an independent predictor in breast cancer patients. Paclitaxel chemotherapy did not benefit patients with low TPM2 expression $(P<0.0001)$. TPM2 knockdown significantly reduced cell sensitivity to paclitaxel. Conclusion: TPM2 is a potential novel tumour suppressor gene in breast cancer. TPM2 is associated with poor survival and chemoresistance to paclitaxel in breast cancer, and TPM2 may represent a promising therapeutic gene target for breast cancer patients with chemoresistance.
\end{abstract}




\section{Introduction}

Breast cancer is the most common malignant tumour in women and one of the most common causes of cancer death worldwide [1]. Although we have greatly improved the treatment for breast cancer patients, some breast tumours become chemoresistant, which dramatically affects patient outcome. Identifying novel genes that are clinically significant for the progression and chemoresistance of breast cancer is critical for evaluating prognosis and treatment of this disease. Although many genes might become prognostic indicators of breast cancer [2,3], some tumour suppressors have been used for molecular targeted therapy [4].

The role of hypoxia in regulating cancer cell invasion and metastasis by influencing the composition of the extracellular matrix has been reported [5]. However, the pathway influencing extracellular matrix remains incompletely explored. DNA hypermethylation is associated with hypoxia and reduces the activity of oxygen-dependent ten-eleven translocation enzymes [6]. Cancer cells from many different human tumours exhibit changes in DNA methylation involving both global hypomethylation and local hypermethylation of CpG-rich gene promoters $[7,8]$. Hypermethylation of promoter CpG islands implies an important mutation-independent mechanism for inactivation of tumour suppressor genes by gene silencing in cancer cells [9], which ultimately contributes to oncogenesis.

Tropomyosins (TPMs) are a family of actin-binding proteins that stabilize and integrate actin microfilaments and are expressed with a high degree of tissue specificity [10-12]. Studies show that several cytoskeleton functions, such as cytokinesis, vesicle transport, cell proliferation, migration and apoptosis, are distinctly influenced and regulated by TPM isoforms $[13,14]$. Moreover, the expression of high molecular weight (HMW) TPMs could reverse stress fibre formation and transform characteristics in most cases, which suggests the tumour suppressor activity of TPM2 and TPM1 [14, 15]. In a recent study, TPM2 was defined as a potential diagnostic biomarker of colorectal cancer [16]. Overexpression of TPM2 suppresses cell proliferation and migration in colorectal cancer cell lines, whereas down-regulated TPM2 is associated with increased tumour proliferation and migration in vitro [17]. However, the role of TPM2 and its regulatory mechanism in breast cancer remain unclear.

Effective chemotherapy can prolong the lives of cancer patients. However, chemoresistance poses a major obstacle to the successful use of drugs in the treatment of cancer [18]. Paclitaxel is an antimitotic agent that binds to microtubules, thereby stabilizing them against depolymerization and inhibiting cell replication by disrupting normal mitotic spindle formation [19]. The effectiveness of paclitaxel in the treatment of many human cancers has been proven [20,21]. TPM2, a cytoskeleton-associated protein, appears to be associated with sensitivity to cisplatin in the breast cancer cell line MCF7 [22]; nonetheless, the influence of TPM2 on paclitaxel remains largely unknown.

In the present study, we first verified TPM2 (TPM2.1 isoform) expression in 48 breast cancer tissues and matched normal tissues. Then, we conducted an immunohistochemical (IHC) study of TPM2 protein expression using a tissue microarray for 410 breast cancer tissues and 185 normal adjacent tissue samples. We then elucidated the mechanism underlying the down-regulation of TPM2 in breast cancer cells in vitro. Finally, we detected the influence of TPM2 on survival and chemotherapy drug sensitivity in breast cancer patients.

\section{Materials and Methods}

\section{Compliance}

With written informed consent forms, tissue samples were obtained from all participating patients, following the relevant protocol approval by the Ethical Committee of Harbin Medical University. The experimental procedure involving patient samples was conducted in accordance with the guideline approved by the Harbin Medical University Institutional Review Board.

\section{KARGER}




\section{Cellular Physiology Cell Physiol Biochem 2018;45:692-705

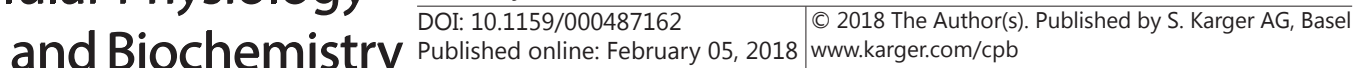 \\ Zhang et al.: The Role of TPM2 in Breast Caner}

\section{Breast cancer patient specimens and clinical features}

All surgical specimens were collected with patient consent and an approved protocol at the Harbin Medical University Cancer Hospital, Harbin, China. Tissues were obtained from 185 patients with normal adjacent tissues and 410 patients with histologically confirmed breast cancer. Fresh cancer tissues and their matched normal tissues from 48 patients were collected and stored at $-80^{\circ} \mathrm{C}$ immediately after resection to extract protein and RNA. The inclusion criteria included the presence of primary, unilateral and operable invasive breast cancer in addition to available data at the initial diagnosis and clinical follow-up. The exclusion criteria included locally advanced disease with recurrent tumour, metastatic disease, other tumours and previous neoadjuvant treatment. Tumour tissues and normal adjacent breast tissues were examined diagnostically by two pathologists.

All patient specimens were subjected to IHC for the oestrogen receptor (ER), progesterone receptor (PR), human epidermal growth factor receptor 2 (HER-2), P53 and Ki67. IHC-positive staining for ER and PR was considered as greater than or equal to $10 \%$ nuclei staining in the invasive component of the tumour [23]. The intensity of the anti-HER2 staining was divided into four grades from $0-3$, such that 0 was negative, 1 was slightly positive, 2 was indeterminate and 3 was positive, as previously reported [24]. Fluorescent in situ hybridization (FISH) was used for all grade 2 samples [25]. Individual samples with $\geq 14 \%$ of Ki67+ tumour cells were considered high-proliferation samples [26]. The invasiveness of the cancers was determined according to the Scarff-Bloom-Richardson system. Tumour-node-metastasis (TNM) stage was evaluated according to the AJCC Cancer Staging Handbook. Individual breast cancer patients were also characterized as molecular subtypes on the basis of previously validated clinicopathological criteria [27].

\section{Follow-up}

All patients were followed up every 3-6 months for the first five years and every 12 months thereafter, while the clinical and pathological records of all patients in the study were also reviewed regularly for analysis. Patients were followed for at least five years at the Harbin Medical University Cancer Hospital until death. The overall survival (OS) was evaluated, and the survival time was defined as the period from the date of diagnosis to the date of death or the study closing date.

DNA extraction, bisulfite sequencing PCR (BSP)

BSP is the standard for DNA methylation detection. The selective chemical reaction of sodium bisulfite with cytosine versus 5 -methylcytosine $(5-\mathrm{MeC})$ residues is the key to the bisulfite protocol for determining DNA methylation. Methylation of a CpG site is indicated by mCpG. Bisulfite modification converts cytosine to uracil in DNA strands. Following PCR amplification, the uracils are amplified as thymines, whereas 5-MeC residues are amplified as cytosines [28].

Genomic DNA was isolated from T47D and MCF7 cells incubated in standard or hypoxic conditions using an AxyPrepTM Multisource Genomic DNA Miniprep Kit (Axygen Scientific, San Francisco, USA) following the manufacturer's instructions. DNA was bisulfite-converted using the EZ DNA MethylationGold kit (Zymo Research, Orange, USA) and amplified using the MegaMix Gold $2 \times$ mastermix and validated primer pairs. A portion of the PCR products (1 to $5 \mu$ l) was cloned into the pTG19-T (Lot: GV6021) vector. Colonies were selected and expanded in liquid culture. Seven validated clones were sequenced at a depth of $\sim 500 \times$ by the GENEray biotechnology library (Shanghai, China). Each CpG island was considered to be methylated when $\geq 4$ of sequenced clones exhibited conservation of the cytosine.

\section{Tissue microarrays and immunohistochemistry staining}

The breast cancer tissue microarray (TMA) for each tissue sample was acquired from paraffin blocks using a thin-walled needle with an inner diameter of $2 \mathrm{~mm}$ to create holes. The IHC detection of TPM2 was performed on each slide. Each section was then incubated with TPM2 (TPM2.1 isoform) rabbit polyclonal antibody solution (OriGene, TA321952).

\section{Evaluation of TPM2 protein expression}

The proportion and staining intensity of TPM2 were evaluated in a series of 10 randomly selected highpower fields (magnification $\mathrm{x} 400$ ), which were considered a representation of the average. The intensity of the IHC staining was graded as 0 (no staining), 1 (weak staining = light yellow), 2 (moderate staining = yellow brown) and 3 (strong staining $=$ brown). The proportion of positively stained tumour cells in a field 


\section{Cellular Physiology Cell Physiol Biochem 2018;45:692-705

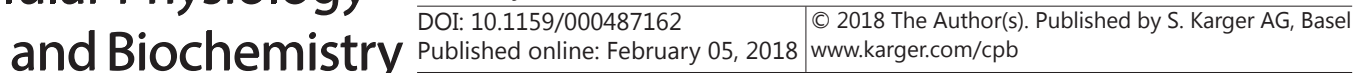 \\ Zhang et al.: The Role of TPM2 in Breast Caner}

was scored as 0 (no positive tumour cells), 1 (fewer than 10\% positive tumour cells), 2 (10-50\% positive tumour cells) and 3 (more than 50\% positive tumour cells) [29]. The staining index (SI) for each sample was obtained by multiplying the intensity and proportion extension values, with a score of less than 4 being classified as low expression.

\section{Cell culture and drug susceptibility experiments}

As control cell lines, MCF7 and T47D were cultured in DMEM medium (Invitrogen, Carlsbad, CA) containing $10 \%$ foetal bovine serum (FBS) at $37^{\circ} \mathrm{C}$ with $5 \% \mathrm{CO}_{2}$ and $21 \% \mathrm{O}_{2}$. To create hypoxia cultures, cells were incubated with an atmosphere of $1.0 \% \mathrm{O}_{2}, 5.0 \% \mathrm{CO}_{2}$ and $94.0 \% \mathrm{~N}_{2}$. The two cell lines were also treated with (30 $\mu \mathrm{M}, 48 \mathrm{~h})$ HIF-1 specific blocker (SC205346; Santa Cruz, CA, USA) in hypoxia culture and (8 $\mu \mathrm{M}, 72$ h) 5-Aza-2'-deoxycytidine (5-Aza-dC; Sigma). UACC-812 and BT-549 were cultured in 1640 medium (Invitrogen, Carlsbad, CA) containing $10 \% \mathrm{FBS}$ at $37^{\circ} \mathrm{C}$ with $5 \% \mathrm{CO}_{2}$. All cell lines were purchased from Chinese Type Culture Collection, Chinese Academy of Sciences. The cells were used for experiments in the logarithmic growth phase.

UACC-812 and BT-549 cells were cultured in a 96-well plate for $24 \mathrm{~h}$, and different concentrations of paclitaxel were added $(0,2,4,8,16,32 \mu \mathrm{g} / \mathrm{L})$ up to half the maximal inhibitory concentration (IC $\left.{ }_{50}\right)$. The solution was mixed to $\mathrm{IC}_{50}$. Transfected cells (scramble and knockdown) were treated with the same concentration of paclitaxel. After treatments, the medium was removed, after which $90 \mu \mathrm{l}$ of RPMI1640 and $10 \mu \mathrm{l}$ of CCK- 8 were added. The plated cells were incubated for $1 \mathrm{~h}$, and then the absorbance at a wavelength of $450 \mathrm{~nm}$ was measured on an automated reader. The inhibition rate (IR) was calculated. IR = [(OD control group - OD experimental group)/OD control group] $\times 100 \%$. We presented the data as the mean \pm standard deviation from five replicate wells per microtiter plate and analysed the IR using a $t$-test. $P$ values $<0.05$ were considered statistically significant.

\section{Cell migration and invasion assay}

The migration and invasion assays were performed in 24-well cell culture inserts (Corning) fitted with a PET membrane ( $8 \mu \mathrm{m}$ pore size). The inserts for invasion assays were coated with $30 \mu \mathrm{L}$ of Matrigel matrix at $37^{\circ} \mathrm{C}$ for $1 \mathrm{~h}$. Transfected cells were plated in medium without serum in the top chamber of a transwell. The bottom chamber contained $600 \mu \mathrm{L}$ RPMI 1640 medium with $10 \% \mathrm{FBS}$. After incubating for $24 \mathrm{~h}$ at $37^{\circ} \mathrm{C}$, the cells that had migrated to the lower surface of the membrane were fixed with $4 \%$ methanal, stained with crystal violet and photographed under a microscope. Cell numbers were counted under a light microscope at $\times 200$ magnification.

\section{Western blot analysis}

The cells were lysed in lysis buffer. Thirty $\mu$ g of sample protein was separated by SDS-PAGE $(10 \%$ gels) and transferred onto a $0.22 \mu \mathrm{m}$ polyvinylidene fluoride (PVDF) membrane. The proteins were probed with TPM2 (OriGene, TA321952), HIF-1 (Wanleibio, China) and MMP2 antibodies (Wanleibio). The bound antibodies were detected using the ECL Western Blotting Detection system. Internal reference of protein loading was assessed using $\beta$-actin (mouse anti- $\beta$-actin, Zhong Shan Xin Qiao, China). Every experiment was conducted three times. We compared the relative grey value between the proteins of interest and the loading control and analysed differences using a $t$-test. $P$ values $<0.05$ were considered statistically significant.

\section{Real-time $q P C R$}

RT-qPCR was performed using the ABI 7500 Fast sequence detection system (Applied Biosystems, Foster City, CA, USA) and FastStart Universal SYBR Green Master (ROX) reagent (Roche Applied Science, Mannheim, Germany) following the manufacturers' instructions. The results of the RT-qPCR experiments were calculated using the $2^{-\Delta \Delta C_{\mathrm{T}}}$ method with minor revision. The main primers in these experiments are listed (for all online suppl. material, see www.karger.com/doi/10.1159/000487162) in Suppl. Table S1.

\section{Public data analysis}

The TPM2 promoter region and first exon region were predicted in

http://genome.ucsc.edu/(UCSC). The CpG island was predicted in

http://www.urogene.org/cgi-bin/methprimer/methprimer.cgi.

The TPM2 expression and DNA methylation data of breast cancer were downloaded from TCGA (https://tcga-data.nci.nih.gov/). 


\section{Cellular Physiology Cell Physiol Biochem 2018;45:692-705 \begin{tabular}{ll|l} 
and Biochemistry Published online: February 05, 2018 & $\begin{array}{l}\text { (c) } 2018 \text { The Author(s). Published by S. Karger AG, Basel } \\
\text { www.karger.com/cpb }\end{array}$ \\
\hline
\end{tabular}

\section{Statistical analysis}

All the analyses were performed using statistical software (SPSS 17.0 for Windows). A $\chi^{2}$ test was used to determine the association between TPM2 expression and patients' clinicopathological features. A $t$-test was used to evaluate the intergroup difference. Survival curves were plotted using the KaplanMeier method, and differences were assessed using the log-rank test. The influence of different variables on survival was assessed using the Cox univariate and multivariate regression analyses. Risk ratios (RR) and their 95\% confidence intervals (CIs) were recorded for each marker. $P$ values $<0.05$ were considered statistically significant.

\section{Results}

TPM2 is down-regulated in breast cancer

TPM2 mRNA expression was examined in tumour and normal tissues using RT-qPCR. The mean TPM2 expression value of mRNA in cancer tissues $(0.266325 \pm 0.46163$, normalized by GAPDH gene expression) was significantly lower than the TPM2 expression value $(0.67564 \pm 0.96855)$ for the corresponding normal tissues (Fig. 1A, $P=0.0005$ ). Western blotting assays showed that TPM2 exhibited low expression in cancer tissues compared with normal tissues (Fig. 1B). TPM2 protein expression in 410 human breast cancer tissues and 185 normal adjacent tissues was examined by IHC. Representative images of the TPM2 IHC staining are shown in Fig. 1C. The protein levels of TPM2 were significantly decreased in cancer tissues relative to normal adjacent tissues (Fig. 1D, $P<0.0001$ ).

Furthermore, data from the TCGA were applied to confirm the mRNA expression of TPM2 by RNA-seq. As expected, mRNA expression of TPM2 in breast cancer tissues was significantly lower than in normal tissues for TCGA (Fig. 1E, $P<0.0001$ ). Collectively, TPM2 expression was down-regulated in breast cancer.

Hypoxia down-regulates the expression of TPM2 in breast cancer

To examine the influence of hypoxia on TPM2 expression, we carried out RT-qPCR analysis of MCF7 and T47D cells incubated in hypoxic conditions for increasing amounts of time. When

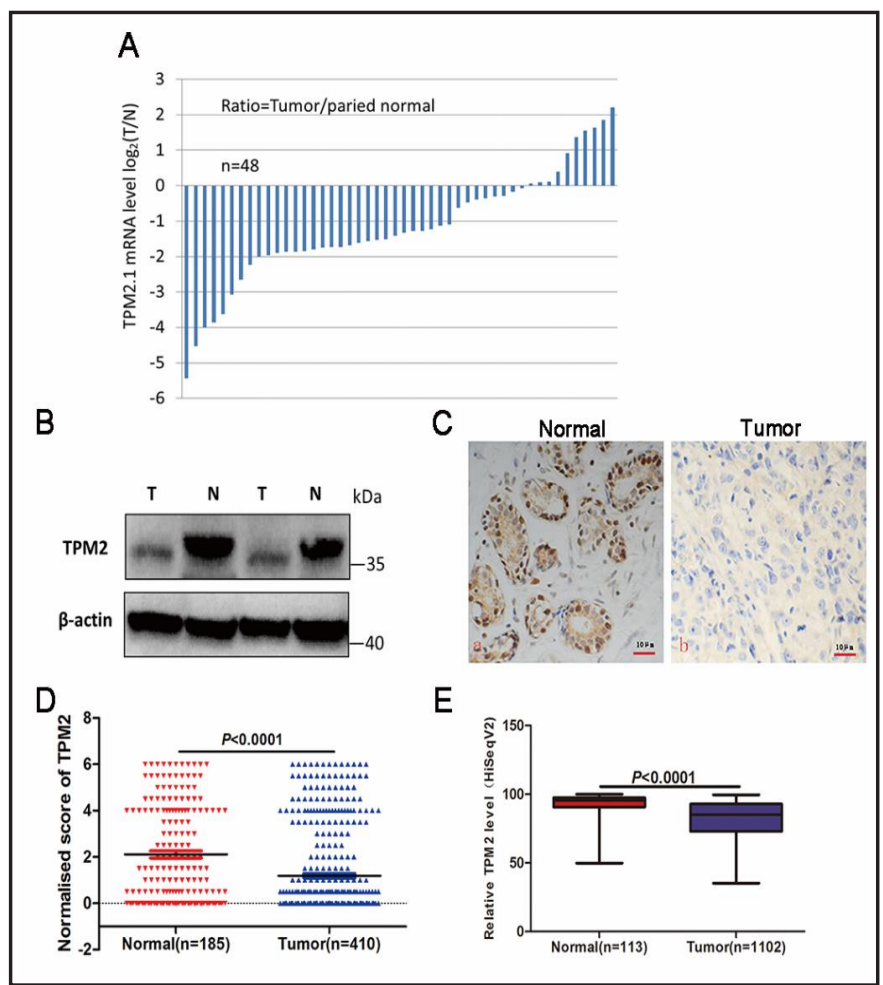

Fig. 1. TPM2 expression is down-regulated in breast cancer. (A) Histogram showing TPM2 mRNA expression in breast cancer. The mRNA expression of TPM2 was calculated by the $2^{-\triangle \Delta \mathrm{CT}_{\mathrm{T}}}$ method, while the relative expression in each patient is presented as the ratio of $\mathrm{T}$ (tumour tissue, $\mathrm{n}=48$ ) $/ \mathrm{N}$ (normal tissue, $\mathrm{n}=48$ ). (B) Representative western blotting analysis of TPM2 expression in breast tissues. The levels of $\beta$-actin were used as an internal control. (C) Representative IHC staining of TPM2 in (a) Normal tissues (n $=185)$ and $(\mathrm{b})$ Tumour tissues $(\mathrm{n}=410)(400 \times)$. The positive TPM2 expression was demonstrated by brown-staining. (D) The expression of TPM2 protein in tumour tissues was significantly lower than that in adjacent normal breast tissues $(\mathrm{P}<0.0001)$. $\mathrm{P}$ values were calculated using Student's t-test. (E) The expression of TPM2 from tumour tissues $(n=1102)$ and normal breast tissues $(\mathrm{n}=113)$ by RNA-seq in TCGA Database $(\mathrm{P}<0.0001)$. 
incubation time was prolonged, TPM2 expression was gradually inhibited (Fig. 2A, 2B). We used mRNA levels of genes encoding fructose-bisphosphate aldolase (ALDOA) and E1B 19K/Bcl2-binding protein Nip3 (BNIP3), two established hypoxia marker genes, as positive controls (Fig. 2C, 2D) [30].

Next, we treated T47D and MCF7 cells in hypoxia with the HIF-1 specific inhibitor, SC205346 $(30 \mu \mathrm{M})$, in order to determine whether blocking HIF-1 would reverse the influence of hypoxia. TPM2 expression was reversed in both the T47D and MCF7 cell lines following treatment with the HIF1 specific blocker for $48 \mathrm{~h}$ (Fig. 2E). We used western blotting to detect the blocking efficiency in these assays (Fig. 2F).

Promoter methylation of TPM2 is responsible for the

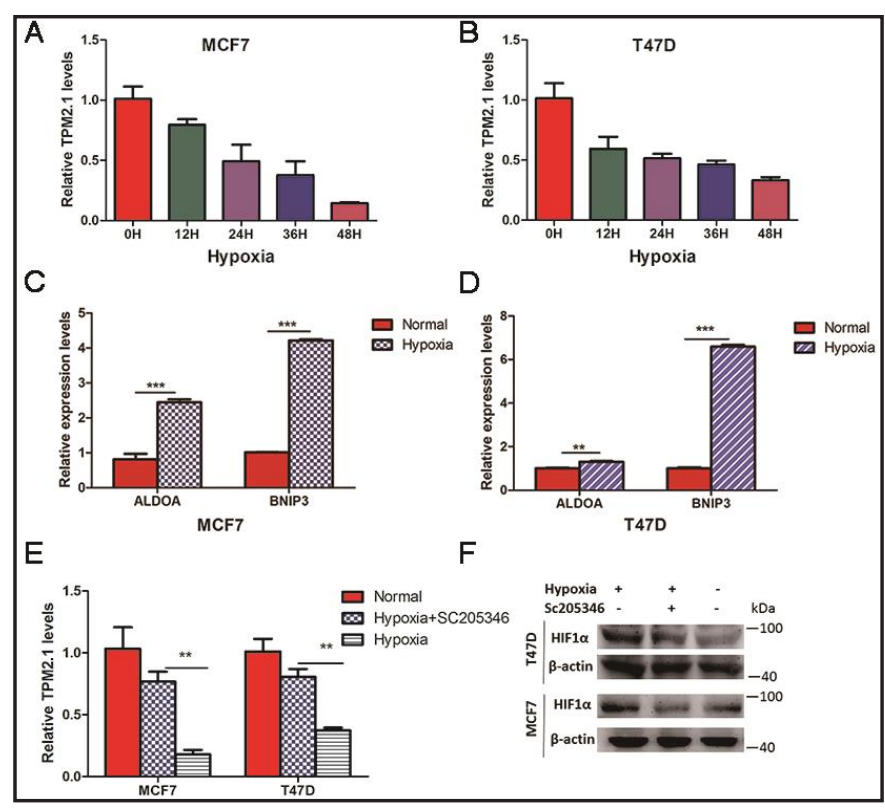

Fig. 2. Hypoxia down-regulates the expression of TPM2 in breast cancer cell lines. (A, B) TPM2 expression was gradually inhibited with increasing incubation times of hypoxia in MCF7 and T47D cell lines. (C, D) ALDOA and BNIP3 were used as positive controls for hypoxia in these assays. (E) TPM2 expression was reversed in both the T47D and MCF7 cell lines following treatment with a HIF1 specific inhibitor (SC205346) for $48 \mathrm{~h}$. (F) Western blotting was used to detect the blocking efficiency of SC205346 in these assays.

\section{low expression of TPM2}

We used BSP to check CPG-rich TPM2 promoter and exon 1 methylation status of the cells incubated in hypoxia for comparison with the cells incubated in standard conditions. The base sequences of the TPM2 promoter and exon 1 are listed, see supplementary material, in Suppl. Table S2. We detected the regions for four sections using BSP. For MCF7 cells, D1 and D3 increased CpG methylation sites in hypoxia. For T47D cells, the D1 section increased one CpG methylation site, while D4 increased six CpG methylation sites in hypoxia (Fig. 3A). When blocking HIF-1 in hypoxia incubation, the changes of promoter and exon 1 methylation status in the two cell lines were reversed (Fig. 3A). We infer that hypoxia may down-regulate the expression of TPM2 by increasing promoter methylation. Microarray data from TCGA hMethyl450 showed that TPM2 expression was gradually down-regulated with an increasing level of methylation status (Fig. 3B). BSP is the standard for DNA methylation analysis, as this method defines the methylation state of individual cytosine residue in the target sequence at single-molecule resolution. We sequenced multiple clones to confirm our results. Methylation status participates in determining the morphological phenotypes of cells. There are cell-type and gene specific mechanisms for regulation of methylation patterns within CpG-rich gene-related regions [31], so methylation sites in T47D and MCF7 appear to be different.

Next, we used the demethylating agent 5-Aza $(8 \mu \mathrm{M})$ to treat T47D and MCF7 cells in order to determine whether blocking promoter methylation resulted in increased TPM2 expression. TPM2 expression increased significantly in both the T47D and MCF7 cell lines following treatment with 5-Aza for $72 \mathrm{~h}$ (Fig. 3C, 3D). We used P16 and CST6 as positive controls in these assays [32, 33].

TPM2 protein expression is associated with breast cancer progression

We investigated the associations between the expression level of TPM2 and various clinicopathological characteristics in breast cancer patients, including age; tumour size; 
Fig. 3. Promoter methylation of TPM2 is responsible for its low expression. (A) Hypoxia increased promoter and exon 1 DNA methylation of TPM2 in breast cancer cells. For MCF7 cells, D1 and D3 sections increased CpG methylation sites. For T47D cells, D1 section

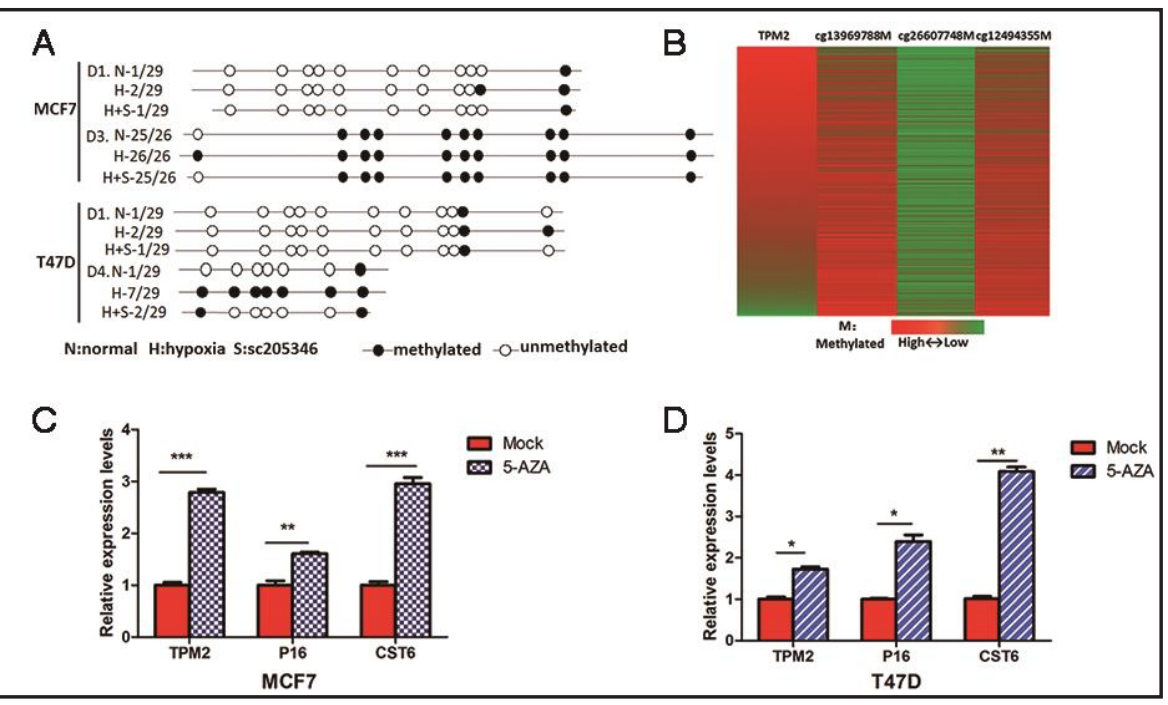
increased one CpG methylation site while D4 increased six CpG methylation sites. Upon blocking HIF-1 in hypoxia incubation, the changes of promoter and exon 1 methylation status in both cell lines were reversed. (B) Microarray data from TCGA hMethyl450 showed that TPM2 expression was gradually down-regulated with increasing levels of methylation status. (C, D) TPM2 expression increased significantly in both MCF7 and T47D cell lines following treatment with 5-Aza $(8 \mu \mathrm{M})$ for $72 \mathrm{~h}$. We used P16 and CST6 as positive controls in these assays. ${ }^{*}, \mathrm{P}<0.05 ;{ }^{* *}, \mathrm{P}<0.01 ;{ }^{* * *}, \mathrm{P}<0.001$.

number and status of lymph node metastasis (LNM); TNM pathologic stage; histological grad; status of ER, PR and HER-2; molecular subtypes; Ki67 and p53 (Table 1). The low-expression TPM2 group exhibited a higher LNM rate $(P=0.031)$, a more advanced TNM stage $(P=0.01)$ and a superior histological grade $(P=0.037)$ compared with the non- or low-expression group. Remarkably, we found a striking discrepancy by comparing LNM number and TPM2 expression $(P<0.0001)$. The frequency and intensity of TPM2 expression were much lower in breast cancer tumours, with a higher degree of invasion and poorer prognosis. In the present study, no such correlations were found between TPM2 and other clinicopathological parameters, including the patient's age, tumour size, Ki67 expression, p53 status, molecular subtype and statuses of ER, PR and HER-2 (Table 1).
Table 1. Clinical characteristics according to the TPM2 expression in 410 breast cancers

\begin{tabular}{|c|c|c|c|}
\hline Characteristics & $\begin{array}{c}\text { High expression } \\
(\mathrm{N}=73)\end{array}$ & $\begin{array}{c}\text { Low expression } \\
(\mathrm{N}=337)\end{array}$ & $P$ value \\
\hline \multicolumn{4}{|l|}{ Age } \\
\hline$<50$ & $45(61.66 \%)$ & 181(53.71\%) & \multirow[t]{2}{*}{0.217} \\
\hline$\geq 50$ & $28(38.34 \%)$ & $156(46.29 \%)$ & \\
\hline \multicolumn{4}{|l|}{ Tumor size } \\
\hline$<2 \mathrm{~cm}$ & $28(38.35 \%)$ & $111(32.94 \%)$ & \multirow{2}{*}{0.375} \\
\hline$\geq 2 \mathrm{~cm}$ & $45(61.65 \%)$ & $226(67.06 \%)$ & \\
\hline Number of lymph node & $1.90 \pm 3.189$ & $4.59 \pm 7.250$ & $<0.0001$ \\
\hline \multicolumn{4}{|l|}{ LNM status } \\
\hline Negative & $42(57.53 \%)$ & $147(43.62 \%)$ & \multirow{2}{*}{0.031} \\
\hline Positive & $31(42.47 \%)$ & $190(56.38 \%)$ & \\
\hline \multicolumn{4}{|l|}{ TNM stage } \\
\hline I ,II & $58(79.45 \%)$ & $215(63.79 \%)$ & \multirow[t]{2}{*}{0.01} \\
\hline III,IV & $15(20.55 \%)$ & $122(36.21 \%)$ & \\
\hline \multicolumn{4}{|l|}{ Histological grade } \\
\hline G1-2 & $32(43.84 \%)$ & $105(31.16 \%)$ & \multirow[t]{2}{*}{0.037} \\
\hline G3 & $41(56.14 \%)$ & $232(68.84 \%)$ & \\
\hline \multicolumn{4}{|l|}{ ER status } \\
\hline Negative & $41(56.16 \%)$ & $186(55.19 \%)$ & \multirow[t]{2}{*}{0.880} \\
\hline Positive & $32(43.84 \%)$ & $151(44.81 \%)$ & \\
\hline \multicolumn{4}{|l|}{ PR status } \\
\hline Negative & $29(\%)$ & $137(\%)$ & \multirow[t]{2}{*}{0.884} \\
\hline Positive & $44(\%)$ & $200(\%)$ & \\
\hline \multicolumn{4}{|l|}{ Her-2 status } \\
\hline Negative & $44(60.27 \%)$ & $221(65.58 \%)$ & \multirow[t]{2}{*}{0.390} \\
\hline Positive & $29(39.73 \%)$ & $116(34.42 \%)$ & \\
\hline \multicolumn{4}{|l|}{ Ki67 status } \\
\hline$<14 \%$ & $43(58.90 \%)$ & $181(53.71 \%)$ & \multirow{2}{*}{0.419} \\
\hline$\geq 14 \%$ & $30(41.10 \%)$ & $156(46.29 \%)$ & \\
\hline \multicolumn{4}{|l|}{ P53 status } \\
\hline Negative & $12(\%)$ & $63(10.13 \%)$ & \multirow[t]{2}{*}{0.651} \\
\hline Positive & $61(\%)$ & $274(26.06 \%)$ & \\
\hline \multicolumn{4}{|l|}{ Subtype } \\
\hline Luminal A & $24(32.87 \%)$ & $110(32.64 \%)$ & \multirow[t]{4}{*}{0.858} \\
\hline Luminal B & $23(31.51 \%)$ & $119(35.31 \%)$ & \\
\hline Her-2 & $13(17.81 \%)$ & $48(14.24 \%)$ & \\
\hline Triple negative & $13(17.81 \%)$ & $60(17.81 \%)$ & \\
\hline
\end{tabular}




\section{Cellular Physiology Cell Physiol Biochem 2018;45:692-705
DOI: 10.1159/000487162 02018 The Author(s). Published by S. Karger AG, Basel and Biochemistry Publishec online: February 05, 2018 (O) 2018 www.karger.coum/cpb

Hypoxia promotes migration and invasion partly by TPM2 down-regulation-mediated changes in matrix metalloproteinase-2(MMP2) expression

RT-qPCR and western blot analysis detected TPM2 mRNA and protein in most of the breast cancer cell lines (Fig. 4A, 4B). To validate the effect of hypoxia and TPM2 expression on metastasis, we chose the previous cell lines T47D and MCF7, as well as UACC-812, which had relatively high expression of TPM2. We knocked down the expression of TPM2 with lentivirus-shRNA and then used western blotting to verify the knockdown efficiency (Fig. 4C).

Hypoxia and TPM2 down-regulation resulted in increased migration and invasion of the three cell lines when compared to controls (Fig. 4D). TPM2 down-regulation-mediated invasion assays were performed in the context of hypoxia, and our findings showed that the migration and invasion were partly enhanced in these conditions (Fig. 4D). To determine the possible mechanism by which hypoxia and TPM2 regulated the migration and invasion of breast cells, western blot analysis was performed to investigate the effects of hypoxia and TPM2 knockdown on MMP2. Both hypoxia and TPM2 knockdown induced marked upregulation of MMP2 expression compared with normoxia. Combining hypoxia with TPM2 knockdown, partly enhanced the up-regulated expression of MMP2 (Fig. 4E). Thus, hypoxia might regulate cell invasiveness partly by TPM2 down-regulation-mediated changes of MMP2 expression.

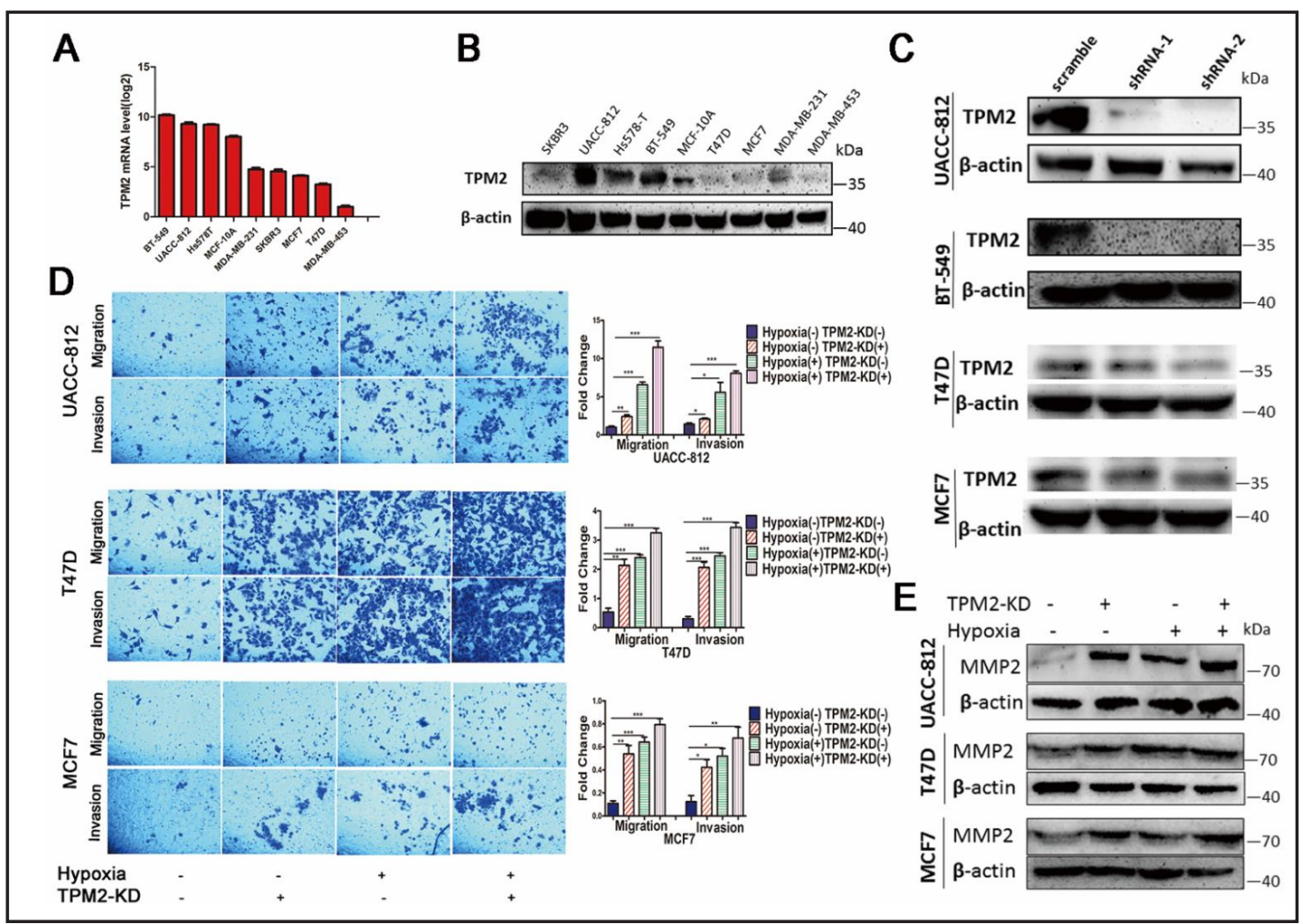

Fig. 4. Hypoxia promotes migration and invasion by TPM2-mediated changes of matrix metalloproteinase-2 (MMP2). (A, B) Expression of TPM2 in 9 breast cancer cell lines was examined by RT-PCR and western blotting. GAPDH and $\beta$-actin were used as internal controls. (C) Western blot detected expression of TPM2 in UACC-812, BT-549, T47D and MCF7 cell lines, which were transfected with scramble(scr) and shRNA. (D) Hypoxia and down-regulated TPM2 expression resulted in increased migration and invasion of UACC-812, T47D and MCF7 cells when compared with controls (scr). (E) Hypoxia and down-regulated TPM2 expression resulted in increased MMP2 expression of UACC-812, T47D and MCF7 cells when compared with controls (scr). *, $\mathrm{P}<0.05 ; * *, \mathrm{P}<0.01 ; * *, \mathrm{P}<0.001$. 


\section{Cellular Physiology Cell Physiol Biochem 2018;45:692-705 and Biochemistry \begin{tabular}{l|l} 
(c) 2018 The Author(s). Published by S. Karger AG, Basel \\
\hline
\end{tabular}

TPM2 expression is related to poor survival in breast cancer patients

We used KaplanMeier OS curves to determine the effects of TPM2 expression on patient survival. The data showed that the 5-year OS rate in patients expressing low levels of TPM2 was significantly lower than in patients with high TPM2 expression $(P=0.028$, Fig. $5 \mathrm{~A})$. Univariate and multivariate survival analyses were performed to evaluate the influence of TPM2 and clinicopathological factors on the prognosis of patients with breast cancer. Univariate hypoxia analyses of OS using a Cox regression analysis identified TPM2 expression $(P=0.033), \quad$ LNM $(P<0.0001), \quad$ PR status $(P=0.014)$ and Ki67 status $(P<0.0001)$ as significant prognostic predictors. Other features had no prognostic value. A multivariate analysis of OS using a Cox regression analysis found that TPM2 expression $(P=0.034)$, Ki67 status $(P=0.012)$, PR status $(P=0.027)$ and LNM $(P<0.0001)$ were independent prognostic factors (Table 2$)$. The present study found that low TPM2 expression is associated with poor outcomes in invasive breast cancer.

\section{Paclitaxel chemotherapy does not benefit patients with low TPM2 expression}

We also used the Kaplan-Meier OS curves to determine the effects of chemotherapy treatment with or without paclitaxel in the TPM2 low-expression group (Fig. 5B). A total of 259 patients out of the 337 patients displayed low TPM2 expression. In the group of patients with low TPM2 expression $(\mathrm{n}=259)$, paclitaxel treatment was less effective than treatment without paclitaxel $(P<0.0001)$. This finding has strong clinical implications and indicates that chemotherapy programmes with paclitaxel may not be effective but harmful in patients with low TPM2 expression.

Down-regulation of TPM2 expression significantly reduced cell sensitivity to paclitaxel

To validate the reliability of the statistical conclusion, we carried out cell experiments. We used the cell lines UACC-812 and BT-549, which had relatively high expression of TPM2, 


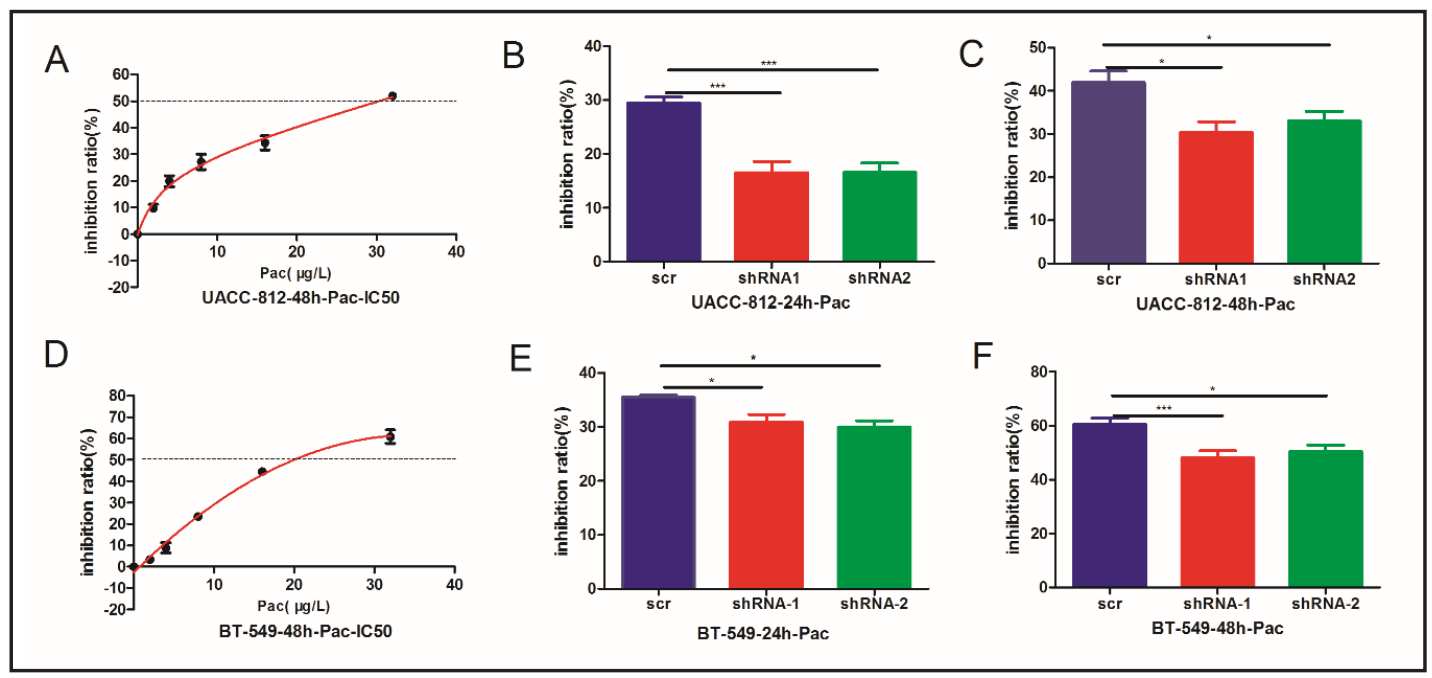

Fig. 6. Down-regulation of TPM2 expression significantly reduced cell sensitivity to paclitaxel.(A) $48 \mathrm{~h} \mathrm{IC50}$ of UACC-812 for paclitaxel. (B) $24 \mathrm{~h}$ inhibition ratio of UACC-812 (scr, shRNA-1, shRNA-2) to paclitaxel. (C) $48 \mathrm{~h}$ inhibition ratio of UACC-812 (scr, shRNA-1, shRNA-2) to paclitaxel. (D) $48 \mathrm{~h}$ IC50 of BT-549 for paclitaxel. (E) $24 \mathrm{~h}$ inhibition ratio of BT-549 (scr, shRNA-1, shRNA-2) to paclitaxel. (F) $48 \mathrm{~h}$ inhibition ratio of BT-549 (scr, shRNA-1, shRNA-2) to paclitaxel. *, $\mathrm{P}<0.05$; **, $\mathrm{P}<0.01$; ***, $\mathrm{P}<0.001$.

to measure the difference of drug sensibility between control and experimental groups. The knockdown efficiency was verified by western blot (Fig. 4C). We determined the IC50 of parental cell lines for paclitaxel. The paclitaxel IC50 concentrations for UACC-812 and BT549 were $31.0 \mu \mathrm{g} / \mathrm{L}$ (Fig. 6A) and $20.8 \mu \mathrm{g} / \mathrm{L}$ (Fig. 6D), respectively. We cultured cell lines (control and experimental groups) with 1640 medium containing the same concentration of paclitaxel and then calculated the growth inhibition ratio of drugs in different cell groups. We found that down-regulated expression of TPM2 significantly reduced cell sensitivity to paclitaxel (Fig. 6B-F).

\section{Discussion}

Altered expression of TPM2 may be common in various tumour types and cancer cell lines [34]; however, the significance of TPM2 in breast cancer has not been investigated. To the best of our knowledge, this is the first study to assess the significance of TPM2 expression in breast cancer, although other studies have also reported the down-regulation of TPM isoforms in a number of transformed cell types and cancers [35]. In this study, we demonstrated that TPM2 expression at both mRNA and protein levels in breast cancer tissues were lower than those in normal tissues. The data from TCGA support this conclusion.

Some studies have suggested that Ras signalling pathways play an important role in TPM down-regulation. Ras signalling consecutively activates Raf/MAPKKK, MEK/MAPK and ERK/MAPK to activate transcription factors that regulate cell growth [36]. Ras-transformed cells usually show down-regulated expression of TPM [37-39]. Some studies have indicated that TPM down-regulation is ERK-dependent in Ras [37] or Jun-transformed cells [34]. By contrast, other studies have suggested that this effect is both MEK- and ERK-independent [38]. In our study, we found that hypoxia affected the methylation status of TPM2 promoters and exon 1 and thus contributed to the down-regulation of TPM2 expression. When blocking HIF-1 during hypoxia incubation, the changes in the two kinds of cells were reversed. Meanwhile, blocking promoter methylation by treating T47D and MCF7 cells with the demethylating agent 5-Aza resulted in increased TPM2 expression. These findings indicate that promoter methylation and exon 1 are responsible for low TPM2 expression. The data from TCGA hMethyl450 showed that down-regulation of TPM2 expression was correlated 
with an increasing level of methylation status. However, the precise mechanisms involved need to be studied further.

Some studies suggest that TPMs are regulators of cell migration and invasion. Migration of cells involves distinct actin filament structures, such as the actin stress fibres, filopodia, actin-meshwork and podosomes [40,41]. However, a number of studies have examined the distribution of TPMs in migration-associated actin filaments, which play an important role in cell migration. Several HMW TPMs are associated with stress fibres [42]. The cancer cell cytoskeleton that is required for many essential functions is disorganized and has a reduced capacity to form specific actin filament populations. This dysfunction is consistent with a reduced expression of TPM isoforms [14]. In the present study, TPM2 expression in cancer cells was correlated with TNM stage $(P=0.01)$ and histological grade $(P=0.037)$. Interestingly, compared with LNM status $(P=0.031)$, the LNM number was markedly correlated with TPM2 expression $(P<0.0001)$. Therefore, lower expression of TPM2 plays an important role in the migration and invasion of breast cancer cells. This is consistent with the results of previous studies on TPM2 $[43,44]$. We performed TPM2 down-regulation mediated migration and invasion experiments for T47D, MCF7 and UACC-812 cells in the context of hypoxia and normoxia. Hypoxia promoted migration and invasion compared with controls. We knocked down TPM2 in breast cancer cell lines and detected the resulting effects on migration and invasion. Down-regulated TPM2 expression resulted in increased migration and invasion of T47D, MCF7 and UACC-812 cells when compared with controls. The ability of migration and invasion were enhanced partly by combining hypoxia with the down-regulation of TPM2. Furthermore, hypoxia and TPM2 knockdown induced marked up-regulation of MMP2 expression compared with normoxia. Thus, hypoxia may regulate cell invasiveness partly by TPM2 down-regulation-mediated changes of MMP2 expression, which is also a new pathway by which hypoxia regulates cancer progression.

We also found that patients with low levels of TPM2 expression had poorer OS $(P=0.028)$, while TPM2 expression was an independent prognostic factor in breast carcinoma. In colorectal cancer, TPM2 was revealed to be a potential diagnostic biomarker in the iTRAQbased quantitative analysis of the cancer-derived secretory proteome [16]. Low expression of TPM2 is associated with RhoA activation and tumour proliferation in colorectal cancer cell lines [17]. These data suggest that low expression of TPM2 has a significant effect on the progression and oncogenesis of human breast cancer. Further studies are needed to confirm these correlations and to reveal the definitive mechanisms involved in breast cancer.

Chemoresistance, which leads to the failure of chemotherapy, is very common in the therapeutic process $[8,45,46]$. Thus, the development of effective chemotherapies is critical. The cytoskeleton plays a central role in many cell processes and thus is an attractive target for chemotherapy $[47,48]$. Specifically, a major chemotherapy target is the microtubule system, which plays an important role in maintaining and regulating cell division [49]. Some studies reported that silencing TPM2 in MCF7 cells affected the stability of lysosomes and the cytoskeleton, thereby enhancing cisplatin-induced cell death [22]. Relevant drugs, such as paclitaxel and vinblastine, are commonly used to stabilize or destabilize microtubules. However, the development of drug resistance can greatly diminish the efficacy of these drugs [50], which can be further hindered by the expression of mutated tubulins [51]. Because paclitaxel is one of the most used chemotherapy drugs in breast cancer, our OS analysis of patients with low TPM2 expression was separated into two groups based on whether or not chemotherapy treatment included paclitaxel. The prognosis of patients with paclitaxel-based chemotherapy treatment was worse than that of patients without paclitaxel treatment $(P<0.0001)$, indicating that breast cancer patients with low TPM2 expression are insensitive to paclitaxel treatment. This result suggested that paclitaxel treatment should not be recommended for these patients. Cellular experiments verified that down-regulated expression of TPM2 significantly reduced the cell inhibition ratio to paclitaxel, which was consistent with statistical results. Paclitaxel resistance associated with the altered expression of specific $\beta$-tubulin isotypes was reported in epithelial ovarian tumours; however, the present study is the first to use TPM2 expression as a predictor of a contraindication to

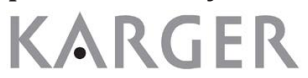


paclitaxel treatment in breast cancer. Effectiveness of chemotherapy is necessary to increase patient survival while decreasing side effects, and our findings may provide the appropriate guidance regarding clinical medication.

The current study is the first to evaluate the expression of TPM2 and its correlation with breast cancer prognosis; however, the current study has some limitations. For example, the follow-up period was not sufficiently long, and the expression status of TPM2 was not analysed in patients with distant metastasis sites. Furthermore, our study was retrospective and included a relatively small number of Chinese patients from a single centre. Nonetheless, our study identified a correlation between TPM2 expression and the survival, prognosis and drug susceptibility of patients with breast carcinoma. Further studies with a larger sample size and multiple centres are needed to verify the functional role and mechanisms of TPM2 in breast cancer.

In summary, low TPM2 expression is frequently found in breast cancer patients where it is associated with metastasis, poor survival and chemoresistance to paclitaxel treatment. Promoter methylation of TPM2, which can be induced by hypoxia, is responsible for low TPM2 expression. Hypoxia may regulate cell invasiveness partly by TPM2 down-regulation mediated changes of MMP2 expression, which is also a new pathway by which hypoxia regulates cancer progression. We conclude that TPM2 is a potential novel tumour suppressor gene in breast cancer. TPM2 is associated with poor survival and chemoresistance to paclitaxel in breast cancer, and TPM2 may represent a promising therapeutic gene target for the breast cancer patients with chemoresistance.

\section{Acknowledgements}

The authors thank the patients for their enthusiastic participation. This work was supported by the Heilongjiang Province Applied Technology Research and Development project (GA13C201); the National Natural Science Foundation of China (81172498/H1622), a specific research fund for the public service sector; the National Health and Family Planning Commission of the People's Republic of China (20140200); and Harbin Medical University Graduate Student Innovation Fund Project (YJSCX2015-31HYD). The funders had no role in the study design, data collection and analysis, decision to publish, or preparation of the manuscript.

\section{Disclosure Statement}

The authors declare no conflicts of interest, financial or otherwise.

\section{References}

-1 Ward EM, DeSantis CE, Lin CC, Kramer JL, Jemal A, Kohler B, Brawley OW, Gansler T: Cancer statistics: Breast cancer in situ. CA Cancer J Clin 2015;65:481-495.

$>2$ He S, He C, Yuan H, Xiong S, Xiao Z, Chen L: The SIRT 3 expression profile is associated with pathological and clinical outcomes in human breast cancer patients. Cell Physiol Biochem 2014;34:2061-2069.

- Z Zhou X, Xie S, Yuan C, Jiang L, Huang X, Li L, Chen Y, Luo L, Zhang J, Wang D, Liu L, Shi W, Han L, Tang N, Ji Y: Lower Expression of SPRY4 Predicts a Poor Prognosis and Regulates Cell Proliferation in Colorectal Cancer. Cell Physiol Biochem 2016;40:1433-1442.

4 Olopade OI, Grushko TA, Nanda R, Huo D: Advances in breast cancer: pathways to personalized medicine. Clin Cancer Res 2008;14:7988-7999.

5 Gilkes DM, Semenza GL, Wirtz D: Hypoxia and the extracellular matrix: drivers of tumour metastasis. Nat Rev Cancer 2014;14:430-439. 


\section{Cellular Physiology Cell Physiol Biochem 2018;45:692-705 \begin{tabular}{l|l|l} 
and Biochemistry Published online: February 05, 2018 & $\begin{array}{l}\text { C } 2018 \text { The Author(s). Published by S. Karger AG, Basel } \\
\text { www.karger.com/cpb }\end{array}$ \\
\hline
\end{tabular} \\ Zhang et al.: The Role of TPM2 in Breast Caner}

6 Thienpont B, Steinbacher J, Zhao H, D’Anna F, Kuchnio A, Ploumakis A, Ghesquiere B, Van Dyck L, Boeckx B, Schoonjans L, Hermans E, Amant F, Kristensen VN, Koh KP, Mazzone M, Coleman ML, Carell T, Carmeliet P, Lambrechts D: Tumour hypoxia causes DNA hypermethylation by reducing TET activity. Nature 2016;537:63-68.

7 Esteller M: Epigenetics in cancer. N Engl J Med 2008;358:1148-1159.

-8 Qiu W, Lin J, Zhu Y, Zhang J, Zeng L, Su M, Tian Y: Kaempferol Modulates DNA Methylation and Downregulates DNMT3B in Bladder Cancer. Cell Physiol Biochem 2017;41:1325-1335.

-9 Herman JG, Baylin SB: Gene silencing in cancer in association with promoter hypermethylation. N Engl J Med 2003;349:2042-2054.

10 Lin JJ, Warren KS, Wamboldt DD, Wang T, Lin JL: Tropomyosin isoforms in nonmuscle cells. Int Rev Cytol 1997;170:1-38.

11 Pawlak G, Helfman DM: Cytoskeletal changes in cell transformation and tumorigenesis. Curr Opin Genet Dev 2001;11:41-47.

12 Pittenger MF, Kazzaz JA, Helfman DM: Functional properties of non-muscle tropomyosin isoforms. Curr Opin Cell Biol 1994;6:96-104.

13 Gunning P, O’Neill G, Hardeman E: Tropomyosin-based regulation of the actin cytoskeleton in time and space. Physiol Rev 2008;88:1-35.

14 O'Neill GM, Stehn J, Gunning PW: Tropomyosins as interpreters of the signalling environment to regulate the local cytoskeleton. Semin Cancer Biol 2008;18:35-44.

15 Raval GN, Bharadwaj S, Levine EA, Willingham MC, Geary RL, Kute T, Prasad GL: Loss of expression of tropomyosin-1, a novel class II tumor suppressor that induces anoikis, in primary breast tumors. Oncogene 2003;22:6194-6203.

16 Ma Y, Xiao T, Xu Q, Shao X, Wang H: iTRAQ-based quantitative analysis of cancer-derived secretory proteome reveals TPM2 as a potential diagnostic biomarker of colorectal cancer. Front Med 2016;10:278285.

17 Cui J, Cai Y, Hu Y, Huang Z, Luo Y, Kaz AM, Yang Z, Chen D, Fan X, Grady WM, Wang J: Epigenetic silencing of TPM2 contributes to colorectal cancer progression upon RhoA activation. Tumour Biol 2016;37:1247712483.

18 Shi C, Qian J, Ma M, Zhang Y, Han B: Notch 3 protein, not its gene polymorphism, is associated with the chemotherapy response and prognosis of advanced NSCLC patients. Cell Physiol Biochem 2014;34:743752.

19 Wallis KT, Azhar S, Rho MB, Lewis SA, Cowan NJ, Murphy DB: The mechanism of equilibrium binding of microtubule-associated protein 2 to microtubules. Binding is a multi-phasic process and exhibits positive cooperativity. J Biol Chem 1993;268:15158-15167.

20 Cohen AL, Ray A, Van Brocklin M, Burnett DM, Bowen RC, Dyess DL, Butler TW, Dumlao T, Khong HT: A phase I trial of azacitidine and nanoparticle albumin bound paclitaxel in patients with advanced or metastatic solid tumors. Oncotarget 2016;10.18632/oncotarget.14183

-21 Murphy WK, Fossella FV, Winn RJ, Shin DM, Hynes HE, Gross HM, Davilla E, Leimert J, Dhingra H, Raber MN, et al.: Phase II study of taxol in patients with untreated advanced non-small-cell lung cancer. J Natl Cancer Inst 1993;85:384-388.

22 Groth-Pedersen L, Aits S, Corcelle-Termeau E, Petersen NH, Nylandsted J, Jaattela M: Identification of cytoskeleton-associated proteins essential for lysosomal stability and survival of human cancer cells. PLoS One 2012; 7:e45381.

23 Matkovic B, Juretic A, Separovic V, Novosel I, Separovic R, Gamulin M, Kruslin B: Immunohistochemical analysis of ER, PR, HER-2, CK 5/6, p63 and EGFR antigen expression in medullary breast cancer. Tumori 2008;94:838-844.

-24 Hsu CY, Ho DM, Yang CF, Lai CR, Yu IT, Chiang H: Interobserver reproducibility of Her-2/neu protein overexpression in invasive breast carcinoma using the DAKO HercepTest. Am J Clin Pathol 2002;118:693698.

-25 Aksoy S, Dizdar O, Harputluoglu H, Altundag K: Demographic, clinical, and pathological characteristics of Turkish triple-negative breast cancer patients: single center experience. Ann Oncol 2007;18:1904-1906.

26 Cheang MC, Chia SK, Voduc D, Gao D, Leung S, Snider J, Watson M, Davies S, Bernard PS, Parker JS, Perou CM, Ellis MJ, Nielsen TO: Ki67 index, HER2 status, and prognosis of patients with luminal B breast cancer. J Natl Cancer Inst 2009;101:736-750. 


\section{Cellular Physiology Cell Physiol Biochem 2018;45:692-705 \begin{tabular}{l|l|l} 
DOI: 10.1159/000487162 & $\begin{array}{l}\text { O } 2018 \text { The Author(s). Published by S. Karger AG, Basel } \\
\text { www.karger.com/cpb }\end{array}$
\end{tabular}}

Zhang et al.: The Role of TPM2 in Breast Caner

27 Goldhirsch A, Winer EP, Coates AS, Gelber RD, Piccart-Gebhart M, Thurlimann B, Senn HJ: Personalizing the treatment of women with early breast cancer: highlights of the St Gallen International Expert Consensus on the Primary Therapy of Early Breast Cancer 2013. Ann Oncol 2013;24:2206-2223.

28 Clark SJ, Statham A, Stirzaker C, Molloy PL, Frommer M: DNA methylation: bisulphite modification and analysis. Nat Protoc 2006;1:2353-2364.

29 Liu T, Zhang X, Shang M, Zhang Y, Xia B, Niu M, Liu Y, Pang D: Dysregulated expression of Slug, vimentin, and E-cadherin correlates with poor clinical outcome in patients with basal-like breast cancer. J Surg Oncol 2013;107:188-194.

-30 Sermeus A, Cosse JP, Crespin M, Mainfroid V, de Longueville F, Ninane N, Raes M, Remacle J, Michiels C: Hypoxia induces protection against etoposide-induced apoptosis: molecular profiling of changes in gene expression and transcription factor activity. Mol Cancer 2008;7:27.

-31 Hu M, Yao J, Cai L, Bachman KE, van den Brule F, Velculescu V, Polyak K: Distinct epigenetic changes in the stromal cells of breast cancers. Nat Genet 2005;37:899-905.

-32 Rivenbark AG, Jones WD, Coleman WB: DNA methylation-dependent silencing of CST6 in human breast cancer cell lines. Lab Invest 2006;86:1233-1242.

33 Herman JG, Graff JR, Myohanen S, Nelkin BD, Baylin SB: Methylation-specific PCR: a novel PCR assay for methylation status of CpG islands. Proc Natl Acad Sci U S A 1996;93:9821-9826.

34 Ljungdahl S, Linder S, Franzen B, Binetruy B, Auer G, Shoshan MC: Down-regulation of tropomyosin-2 expression in c-Jun-transformed rat fibroblasts involves induction of a MEK1-dependent autocrine loop. Cell Growth Differ 1998;9:565-573.

-35 Franzen B, Linder S, Uryu K, Alaiya AA, Hirano T, Kato H, Auer G: Expression of tropomyosin isoforms in benign and malignant human breast lesions. Br J Cancer 1996;73:909-913.

-36 Schubbert S, Shannon K, Bollag G: Hyperactive Ras in developmental disorders and cancer. Nat Rev Cancer 2007;7:295-308.

37 Shields JM, Mehta H, Pruitt K, Der CJ: Opposing Roles of the Extracellular Signal-Regulated Kinase and p38 Mitogen-Activated Protein Kinase Cascades in Ras-Mediated Downregulation of Tropomyosin. Molecular and Cellular Biology 2002;22:2304-2317.

-38 Kim PN, Jonasch E, Mosterman BC, Mier JW, Janssen RA: Radicicol suppresses transformation and restores tropomyosin-2 expression in both ras- and MEK-transformed cells without inhibiting the Raf/MEK/ERK signaling cascade. Cell Growth Differ 2001;12:543-550.

-39 Janssen RA, Veenstra KG, Jonasch P, Jonasch E, Mier JW: Ras- and Raf-induced down-modulation of nonmuscle tropomyosin are MEK-independent. J Biol Chem 1998;273:32182-32186.

40 Gimona M, Buccione R: Adhesions that mediate invasion. Int J Biochem Cell Biol 2006;38:1875-1892.

-41 Ridley AJ, Schwartz MA, Burridge K, Firtel RA, Ginsberg MH, Borisy G, Parsons JT, Horwitz AR: Cell migration: integrating signals from front to back. Science 2003;302:1704-1709.

42 Schevzov G, Vrhovski B, Bryce NS, Elmir S, Qiu MR, O’Neill G M, Yang N, Verrills NM, Kavallaris M, Gunning PW: Tissue-specific tropomyosin isoform composition. J Histochem Cytochem 2005;53:557-570.

-43 Janssen RA, Mier JW: Tropomyosin-2 cDNA lacking the 3' untranslated region riboregulator induces growth inhibition of v-Ki-ras-transformed fibroblasts. Mol Biol Cell 1997;8:897-908.

44 Gimona M, Kazzaz JA, Helfman DM: Forced expression of tropomyosin 2 or 3 in v-Ki-ras-transformed fibroblasts results in distinct phenotypic effects. Proc Natl Acad Sci U S A 1996;93:9618-9623.

45 Tian Z, Yao G, Song H, Zhou Y, Geng J: IGF2R expression is associated with the chemotherapy response and prognosis of patients with advanced NSCLC. Cell Physiol Biochem 2014;34:1578-1588.

46 Chen Y, Gao Y, Zhang K, Li C, Pan Y, Chen J, Wang R, Chen L: MicroRNAs as Regulators of Cisplatin Resistance in Lung Cancer. Cell Physiol Biochem 2015;37:1869-1880.

47 Giganti A, Friederich E: The actin cytoskeleton as a therapeutic target: state of the art and future directions. Prog Cell Cycle Res 2003;5:511-525.

48 Rao J, Li N: Microfilament actin remodeling as a potential target for cancer drug development. Curr Cancer Drug Targets 2004; 4:345-354.

49 Jordan MA, Wilson L: Microtubules and actin filaments: dynamic targets for cancer chemotherapy. Curr Opin Cell Biol 1998;10:123-130.

-50 Robinson DN, Spudich JA: Mechanics and regulation of cytokinesis. Curr Opin Cell Biol 2004;16:182-188.

-51 Chauncey TR: Drug resistance mechanisms in acute leukemia. Curr Opin Oncol 2001;13:21-26. 